\title{
Overdoped end of the cuprate phase diagram
}

\author{
Thomas A. Maier $\odot,{ }^{1,2}$ Seher Karakuzu, ${ }^{2}$ and Douglas J. Scalapino ${ }^{3}$ \\ ${ }^{1}$ Computational Sciences and Engineering Division, Oak Ridge National Laboratory, Oak Ridge, Tennessee 37831-6164, USA \\ ${ }^{2}$ Center for Nanophase Materials Sciences, Oak Ridge National Laboratory, Oak Ridge, Tennessee 37831-6164, USA \\ ${ }^{3}$ Department of Physics, University of California, Santa Barbara, California 93106-9530, USA
}

(Received 24 April 2020; revised 30 June 2020; accepted 2 July 2020; published 23 July 2020)

\begin{abstract}
Studying the disappearance of superconductivity at the end of the overdoped region of the cuprate phase diagram offers a different approach for investigating the interaction which is responsible for pairing in these materials. In the underdoped region this question is complicated by the presence of charge and stripe ordered phases as well as the pseudogap. In the overdoped region the situation appears simpler with only a normal phase, a superconducting phase, and impurity scattering. Here, for the overdoped region, we report the results of a combined dynamic cluster approximation (DCA) and a weak Born impurity scattering calculation for a $t-t^{\prime}-U$ Hubbard model. We find that a decrease in the $d$-wave pairing strength of the two-particle scattering vertex is closely coupled to changes in the momentum and frequency structure of the magnetic spin fluctuations as the system is overdoped. Treating the impurity scattering within a disordered BCS $d$-wave approximation, we see how the combined effects of the decreasing $d$-wave pairing strength and weak impurity scattering lead to the end of the $T_{c}$ dome.
\end{abstract}

DOI: 10.1103/PhysRevResearch.2.033132

\section{INTRODUCTION}

In the overdoped region of the cuprate phase diagram the normal phase exhibits properties similar to those of a strongly correlated Fermi liquid [1-3]. The pseudogap as well as the charge and stripe ordered phases, which compete or coexist with superconductivity at lower doping, are absent [4]. This is a region in which the results of numerical calculations are expected to be less sensitive to boundary conditions and lattice size effects. Previous dynamic cluster approximation (DCA) calculations for a single-band Hubbard model have found that in the overdoped region the superconducting transition temperature $T_{c}(x)$ falls as the density $x$ of doped holes per site increases [5-7]. Moreover, the $d$-wave pairing strength was shown to decrease despite the persistence of magnetic spin fluctuations in determinant quantum Monte Carlo calculations of the overdoped Hubbard model [8]. Here we expand on this research by studying the relation between the decrease in the pairing interaction and the magnetic fluctuations in more detail and specifically its relation to a Lifshitz transition in the Fermi surface topology, and we examine the additional effect of impurity scattering on the decrease of $T_{c}$. In particular, we use DCA calculations [9] to study the decrease in the strength of the $d$-wave pairing interaction for a $t-t^{\prime}-U$ Hubbard model in the overdoped regime as the hole density $x$ increases [10]. Then, including impurity scattering within a

Published by the American Physical Society under the terms of the Creative Commons Attribution 4.0 International license. Further distribution of this work must maintain attribution to the author(s) and the published article's title, journal citation, and DOI. disordered Bardeen-Cooper-Schrieffer (BCS) $d$-wave approximation [11], we examine the end of the $T_{c}$ dome.

The Hubbard model we will study has a near-neighbor hopping $t$, a next-near-neighbor hopping $t^{\prime}$, and an on-site Coulomb interaction $U$. Its Hamiltonian reads

$$
\begin{aligned}
H= & -t \sum_{\langle i, j\rangle \sigma} c_{i \sigma}^{\dagger} c_{j \sigma}-t^{\prime} \sum_{\langle\langle i, j\rangle\rangle \sigma} c_{i \sigma}^{\dagger} c_{j \sigma} \\
& +U \sum_{i} n_{i \uparrow} n_{i \downarrow}-\mu \sum_{i \sigma} n_{i \sigma} .
\end{aligned}
$$

The tight-binding parameters give rise to a band structure $\varepsilon_{k}=-2 t\left(\cos k_{x}+\cos k_{y}\right)-4 t^{\prime} \cos k_{x} \cos k_{y}$ and $\mu$ controls the filling, which we will measure in terms of the density of holes $x$ away from half-filling. The results we present in the following for $t^{\prime} / t=-0.25$ and $U / t=7.0$ were obtained from DCA calculations using a 12 -site cluster (Fig. 2 in Ref. [9]) and a continuous-time auxiliary field quantum Monte Carlo algorithm $[12,13]$ to solve the DCA cluster problem.

In Fig. 1 we show a section of a $t^{\prime} / t-x$ phase diagram for $U / t=7$ based upon DCA calculations of the single-particle spectral weight by Wu et al. [14]. The solid (black) curve marks a Lifshitz transition at which the topology of the Fermi surface changes from electronlike around the origin $(0,0)$ of the Brillouin zone as the hole doping decreases. The dashed (blue) curve in Fig. 1 marks the end of the pseudogap (PG) regime. Similar to the cuprates, as discussed by DoironLeyraud et al. [4], the simulations find that a PG does not open on an electronlike Fermi surface (FS), confining the PG to a region of the $t^{\prime} / t-x$ phase diagram in which $x$ is less than the curve marking the Lifshitz doping. However, for larger values of $\left|t^{\prime} / t\right|$ there is a range of dopings below the Lifshitz doping in which the PG is also absent $[4,14]$. It is in this region, along the dash-dotted $t^{\prime} / t=-0.25$ (red) line that we will examine 


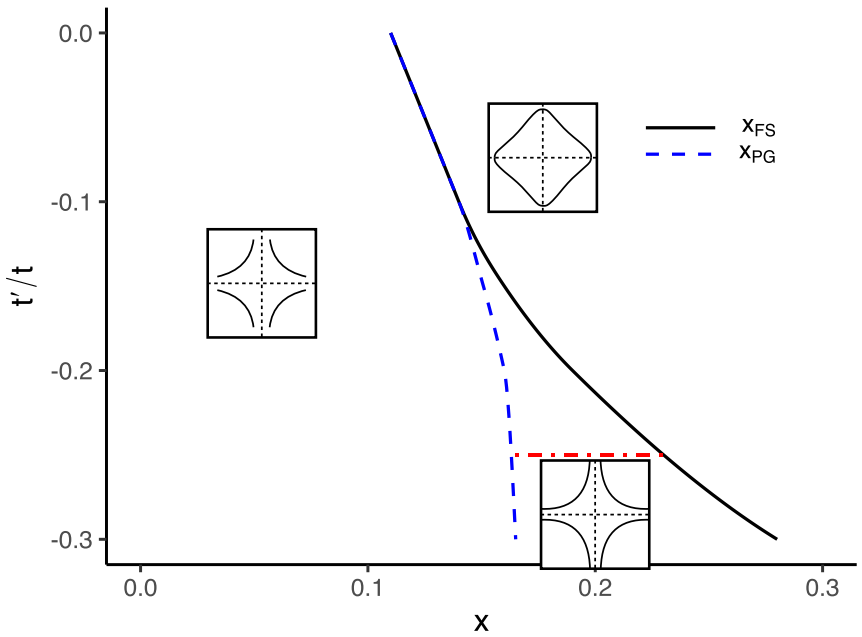

FIG. 1. A part of the $t^{\prime} / t-x$ phase diagram based on DCA calculations [14]. The solid (black) curve marks a Lifshitz transition and the dashed (blue) curve, which separates from the Lifshitz curve at larger doping, marks the end of the pseudogap region. The region to the right of the Lifshitz curve has an electronlike FS around the origin of the Brillouin zone and the region between the Lifshitz curve and the dashed blue curve has a hole like FS around the $(\pi, \pi)$ point. Here we will study the decrease of the $d$-wave pairing strength and the end of the $T_{c}$ dome for $t^{\prime} / t=-0.25$ as the hole doping increases along the dash-dotted (red) line.

the strength of the $d$-wave pairing and the end of the $T_{c}$ dome as the doping is increased.

In the following, we begin by calculating the change in the momentum and frequency dependence of the spin susceptibility for $t / t=-0.25$ as the doping $x$ increases. We observe that the spectral weight at large momentum transfer is significantly reduced as one moves to larger dopings along the dash-dotted (red) line in Fig. 1. Following this, we analyze the particle-particle Bethe-Salpeter vertex and find that the $d$-wave pairing strength also decreases as the doping $x$ increases. Then, using the DCA results and treating the impurity scattering associated with the doping with in a disordered BCS $d$-wave approximation, we determine $T_{c}(x)$ at the overdoped end of the phase diagram.

\section{SPIN SUSCEPTIBILITY AND $d$-WAVE PAIRING STRENGTH}

In Fig. 2 we have plotted the spin susceptibility $\chi(\boldsymbol{q})$ at $T=0.1 t$ for dopings $x=0.15$ and $x=0.25$. At the larger doping, the susceptibility at large momentum transfer $\boldsymbol{q}=$ $(\pi, \pi)$ is reduced and the ferromagnetic (FM) spin susceptibility at small momentum transfer is increased. A similar behavior as the doping approaches the Lifshitz curve has been found in the Lindhard susceptibility [15]. However, as previously noted [8], a significant response also remains at intermediate values of momentum transfer $\boldsymbol{q}=(\pi / 2, \pi / 2)$ and $\boldsymbol{q}=(\pi, 0)$. Further insight into the evolution of the spin fluctuations with doping is illustrated in Fig. 3 where we have plotted the spin-fluctuation spectral weight $-\frac{1}{\pi} \operatorname{Im} \chi(\boldsymbol{q}, \omega)$ at different momentum transfers for various dopings. These results were obtained from a maximum entropy analytic
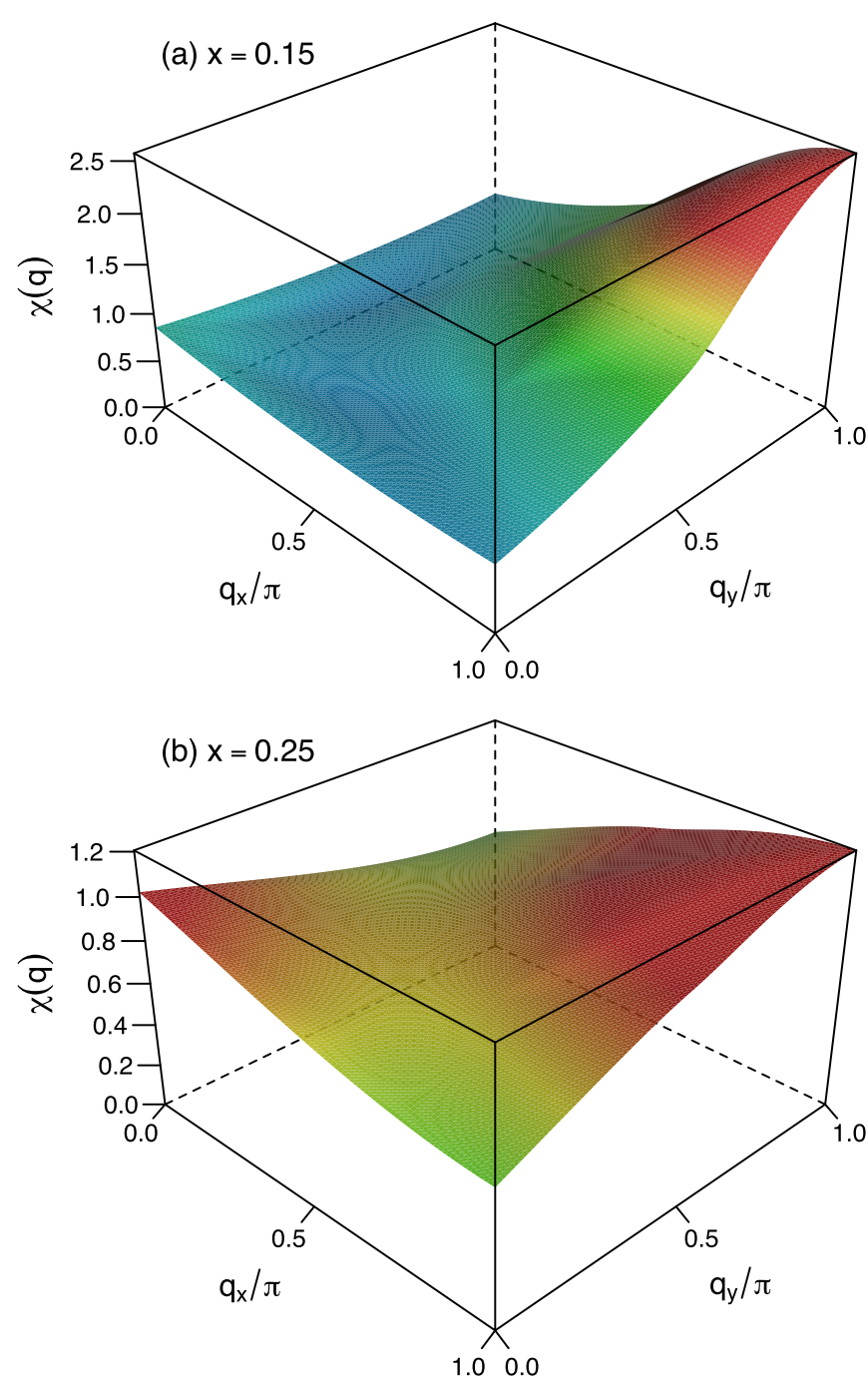

FIG. 2. The spin susceptibility $\chi(\boldsymbol{q})$ at $T=0.1 t$ for (a) $x=0.15$ and (b) 0.25 . As the doping increases the antiferromagnetic response weakens and there is an increase in the ferromagnetic response.

continuation of the DCA imaginary time data. At large momentum transfer $\boldsymbol{q}=(\pi, \pi)$, one sees that the spin-fluctuation spectral weight is significantly reduced with doping, while at intermediate values of momentum transfers spin fluctuations remain. For example, at the Brillouin zone boundary $\boldsymbol{q}=(\pi, 0)$, the magnetic excitations are relatively unchanged with doping. This type of behavior has been seen in inelastic neutron scattering and resonant inelastic x-ray scattering experiments [16-19].

Next we turn to the strength of the pairing interaction in the overdoped region. The particle-particle Bethe-Salpeter equation is

$$
\lambda_{\alpha} \phi_{\alpha}(k)=-\frac{T}{N} \sum_{k^{\prime}} \Gamma\left(k, k^{\prime}\right) G\left(k^{\prime}\right) G\left(-k^{\prime}\right) \phi_{\alpha}\left(k^{\prime}\right) .
$$

Here $G$ is the single-particle propagator and $\Gamma$ is the irreducible particle-particle scattering vertex and we have used $k=\left(\boldsymbol{k}, \omega_{n}\right)$. At the superconducting transition temperature $T_{c}$ the leading eigenvalue of Eq. (2) goes to 1. For the doped Hubbard model the eigenfunction $\phi_{d}\left(\boldsymbol{k}, \omega_{n}\right)$ with the leading 
(a) $q=(0,0)$

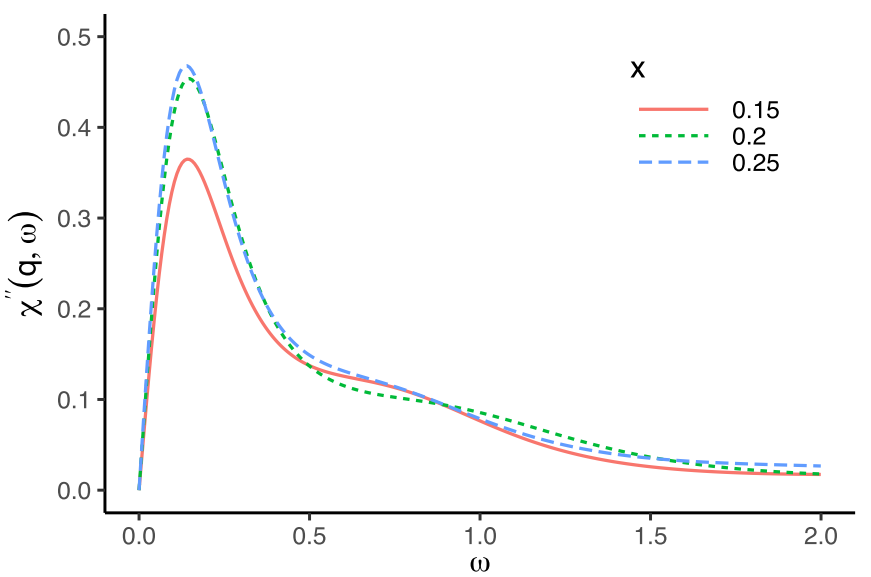

(b) $q=(\pi, 0)$

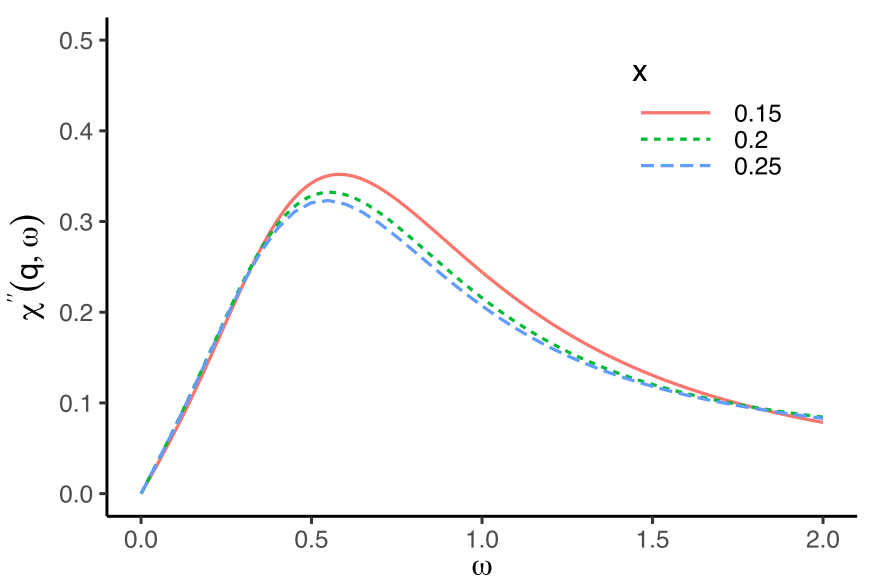

(c) $q=(\pi, \pi)$

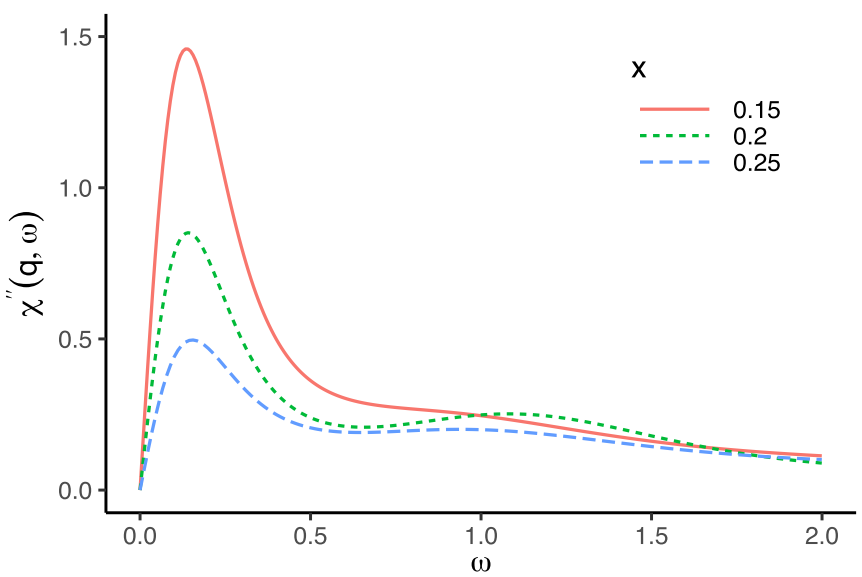

FIG. 3. The imaginary part of the DCA cluster spin susceptibility $\chi^{\prime \prime}(\boldsymbol{q}, \omega)$ vs $\omega$ for various cluster momenta and dopings $x=0.15$ (solid red), 0.2 (short-dashed green), and 0.25 (longdashed blue). The spin fluctuation spectral weight at large (AF) momenta is reduced as the doping increases while the longwavelength (FM) spectral weight increases. For intermediate momenta $\boldsymbol{q}=(\pi, 0)$, the high-energy spin-fluctuation spectral weight remains. eigenvalue has $d$-wave symmetry. At a doping $x=0.15$ the DCA calculations give $\lambda_{d}\left(T_{c}\right)=1$ with $T_{c} / t=0.027$. For a near-neighbor hopping $t=0.2 \mathrm{eV}$, this would correspond to a transition temperature $T_{c} \sim 65 \mathrm{~K}$. Here we are interested in what happens to the strength of the pairing interaction as $x$ increases and the system is overdoped.

Multiplying Eq. (2) by $\phi_{d}\left(\boldsymbol{k}, \omega_{n}\right)$ and summing over $\left(\boldsymbol{k}, \omega_{n}\right)$ one obtains the following expression for $\lambda_{d}$ :

$$
\lambda_{d}=\frac{-\frac{T^{2}}{N^{2}} \sum_{k, k^{\prime}} \phi_{d}(k) \Gamma\left(k, k^{\prime}\right) G\left(k^{\prime}\right) G\left(-k^{\prime}\right) \phi_{d}\left(k^{\prime}\right)}{\frac{T}{N} \sum_{k} \phi_{d}^{2}(k)} .
$$

Then inserting a complete set of states between the vertex $\Gamma$ and the $G G$ propagators and assuming that the leading $d$-wave eigenvalue is dominant, one obtains the separable approximation

$$
\lambda_{d}(T) \simeq V_{d}(T) P_{d 0}(T)
$$

with the strength of the pairing interaction

$$
V_{d}(T)=-\frac{\sum_{k} \sum_{k^{\prime}} \phi_{d}(k) \Gamma\left(k, k^{\prime}\right) \phi_{d}\left(k^{\prime}\right)}{\left[\sum_{k} \phi_{d}^{2}(k)\right]^{2}}
$$

and the noninteracting but dressed two-particle pairfield susceptibility

$$
P_{d 0}(T)=\frac{T}{N} \sum_{k} \phi_{d}^{2}(k) G(k) G(-k) .
$$

In evaluating these expressions we will approximate the $d$ wave eigenfunction

$$
\phi_{d}\left(\boldsymbol{k}, \omega_{n}\right) \sim\left(\cos k_{x}-\cos k_{y}\right) \frac{(\pi T)^{2}+\omega_{c}^{2}}{\omega_{n}^{2}+\omega_{c}^{2}}
$$

with $\omega_{c}=t$. This form provides a reasonable approximation and is less noisy than using the DCA eigenfunction $\phi\left(\boldsymbol{k}, \omega_{n}\right)$.

To check the validity of the separable approximation for $\lambda_{d}(T)$ given by Eq. (4) we have plotted $\lambda_{d}(T)$ and the product $V_{d}(T) P_{d 0}(T)$ versus $T$ in Fig. 4 for different dopings. The close agreement between $\lambda_{d}(T)$ and $V_{d}(T) P_{d 0}(T)$ seen in Fig. 4 arises from the fact that, while there are other singlet eigenstates of the Bethe-Salpeter equation such as extended $s$-wave and odd frequency $p$-waves, the singlet channel is dominated by the $d$-wave eigenfunction. Thus $V_{d}$ provides a measure of the $d$-wave pairing strength associated with the two-particle scattering vertex $\Gamma\left(\boldsymbol{k}, \omega_{n}, \boldsymbol{k}^{\prime}, \omega_{n^{\prime}}\right)$. Results for $V_{d}(x)$ at a low temperature $T=0.08 t$ are shown in Fig. 5(a). Here one sees the decrease of the $d$-wave coupling strength as the hole doping is increased.

In spin-fluctuation theories of the pairing interaction, a measure of the strength of the $d$-wave pairing interaction is given by

$$
V_{d}^{\mathrm{SF}}=\frac{3 \bar{U}^{2}}{2} \frac{1}{N} \sum_{\boldsymbol{q}} \int_{0}^{\infty} \frac{d \omega}{\pi} \frac{\operatorname{Im} \chi(\boldsymbol{q}, \omega)}{\omega} \cos q_{x} .
$$

Using the DCA results for the cluster spin susceptibility $\chi(\boldsymbol{q}, \omega)$ and replacing $\bar{U}$ by $U / 2$, as found in previous DCA studies [20], results for $V_{d}^{\mathrm{SF}}(x)$ versus $x$ at $T=0.08 t$ are 


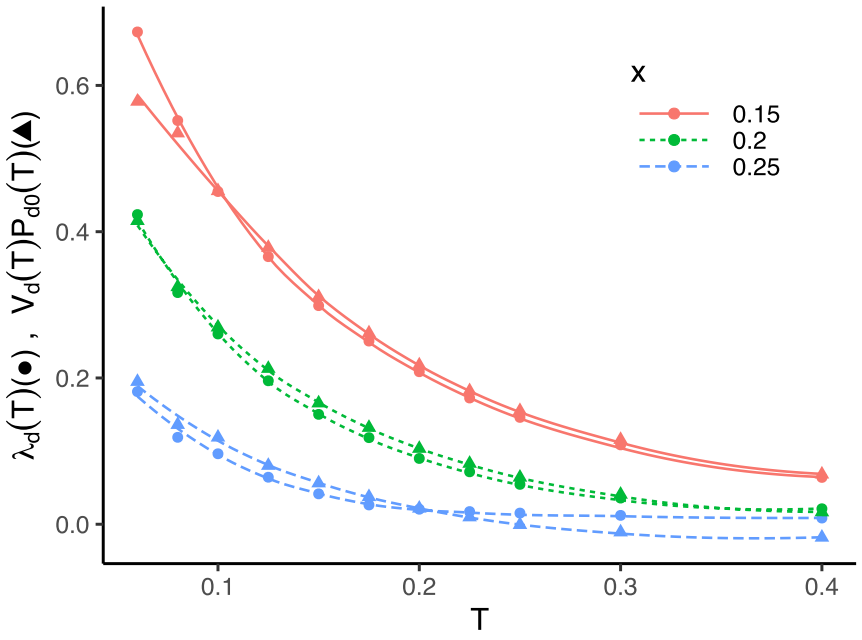

FIG. 4. A comparison of the $d$-wave eigenvalue $\lambda_{d}(T)(\bullet)$ of the Bethe-Salpeter equation (2) with the separable approximation $P_{d 0}(T) V_{d}(T)(\boldsymbol{\Lambda})$ given by Eqs. (5) and (6) for several values of the doping $x$. The quality of the fit reflects the dominance of the leading $d$-wave eigenvalue.

plotted in Fig. 5(b). The change in $\chi(\boldsymbol{q})$ shown in Fig. 2 and the shift of the spin-fluctuation spectral weight with doping shown in Fig. 3 are reflected in the decrease in $V_{d}^{\mathrm{SF}}$ as the doping increases.

The decrease in the large momentum spin-fluctuation spectral weight with doping shown in Fig. 3(c) and its correlation with the decrease in $T_{c}$ in the overdoped regime has been reported for a number of cuprates [18,21-23]. This has been used as circumstantial evidence that there is a close relationship between antiferromagnetic (AF) spin fluctuations and superconductivity. Here, from a direct comparison of the $d$-wave spin-fluctuation pairing strength $V_{d}^{S F}(x)$ with the pairing strength $V_{d}(x)$ obtained from the particle-particle BetheSalpeter vertex $\Gamma(x)$, shown in Fig. 5, we have evidence for the Hubbard model that the decrease in the AF spin fluctuation spectral weight causes a decrease in the $d$-wave pairing (a)

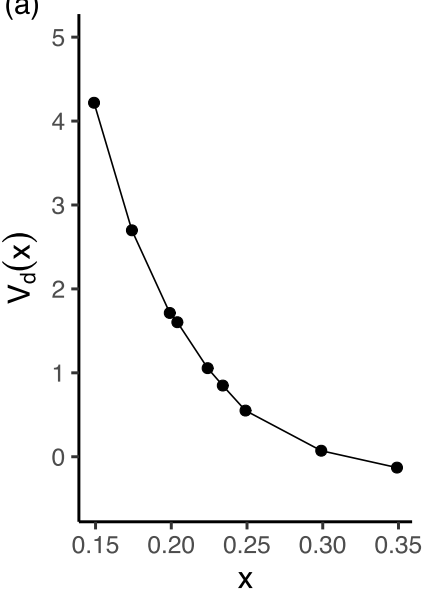

(b)

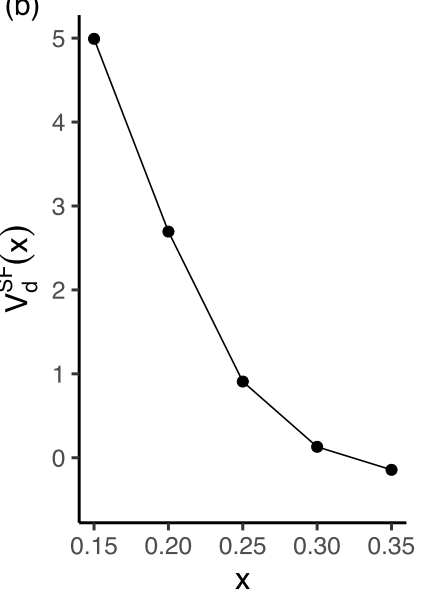

FIG. 5. (a) The $d$-wave pairing strength $V_{d}(x)$, Eq. (5), at $T=$ $0.08 t$ obtained from the two-particle scattering vertex $\Gamma$ plotted versus the doping $x$. (b) The $d$-wave spin-fluctuation pairing strength, $V_{d}^{\mathrm{SF}}(x)$ Eq. (8), at $T=0.08 t$ versus $x$.

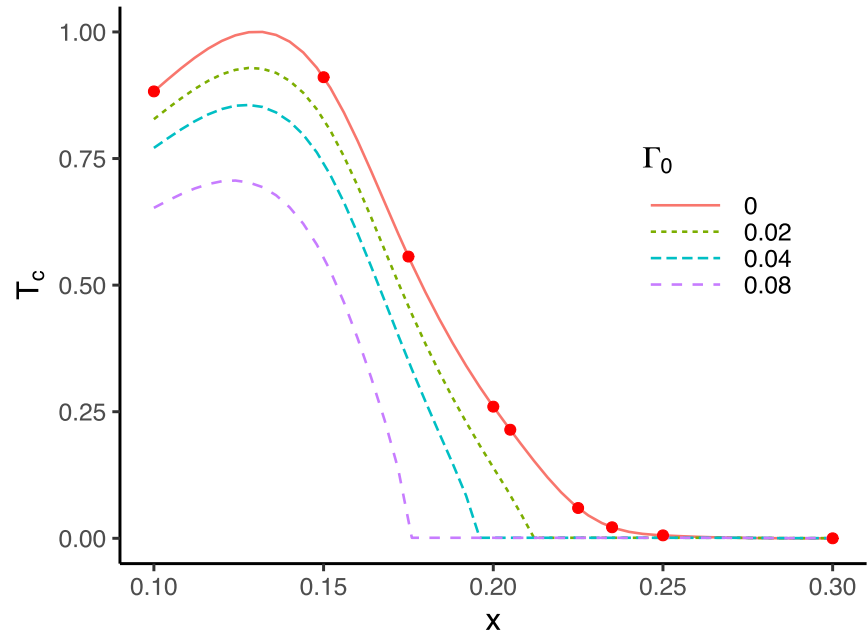

FIG. 6. The superconducting transition temperature $T_{c 0}(x)$ (solid red dots) for the pure system determined from an extrapolation of the Bethe-Salpeter eigenvalue $\lambda_{d}(T)$ to 1 for specific dopings. The red solid curve is a fit to these points. The additional dashed $T_{c}(x)$ curves are solutions of the AG equation for different impurity scattering strengths $\Gamma_{0}$. The transition temperatures are normalized by the maximum $T_{c 0}$ value.

strength. To the extent that the Hubbard model exhibits many of the properties seen in the cuprate superconductors, this supports the idea that large momentum AF spin fluctuations mediate the pairing in these materials.

\section{THE END OF THE $T_{c}$ DOME}

The end behavior of the $T_{c}$ dome involves the effects of impurities. Here we have in mind a situation in which the impurity dopants lie off of the $\mathrm{CuO}_{2}$ plane, adding $x$ holes and giving rise to weak Born impurity scattering. Within the framework of a fluctuation exchange approximation, Kudo and Yamada [24] found that the reduction of the BetheSalpeter eigenvalue associated with the decrease in strength of the pairing interaction caused by impurity scattering is approximately offset by the increase of the spectral weight of the single-particle propagator, leaving pair breaking as the dominant effect of the impurity scattering. We will assume that this is also the case here and use the Abrikosov-Gorkov [25] expression for the superconducting transition temperature given by

$$
\ln \left(\frac{T_{c 0}(x)}{T_{c}(x)}\right)=\psi\left(\frac{1}{2}+\frac{\Gamma(x)}{2 \pi T_{c}(x)}\right)-\psi(1 / 2) .
$$

Here $T_{c 0}(x)$ is the putative superconducting transition temperature of the doped system without impurity scattering obtained by extrapolating the eigenvalue of the Bethe-Salpeter equation $\lambda_{d}\left[T_{c 0}(x)\right]$ to 1 . Assuming that the impurity dopants lie out of plane, $\Gamma(x)$ is the normal-state Born impurity scattering rate, which we take proportional to $x$,

$$
\Gamma(x)=\Gamma_{0} x,
$$

and $\psi$ is the digamma function. Results for $T_{c}(x)$ versus $x$ for various values of the scattering rate $\Gamma_{0}$ per doped hole are shown in Fig. 6. 
Here $T_{c 0}(x)$ vanishes with an essential singularity $\exp \left[-t / V_{d}(x)\right]$ at an end point where $V_{d}(x)$ goes to zero. In the dirty $d$-wave theory, $T_{c}(x)$ approaches the end point $x_{0}$ as $\left(x-x_{0}\right)^{1 / 2}$ with $x_{0}$ determined by

$$
T_{c 0}\left(x_{0}\right) / \Gamma\left(x_{0}\right)=2 \gamma / \pi
$$

with $\gamma \sim 1.78$.

\section{DISCUSSION AND CONCLUSION}

We have used a combined DCA and weak Born impurity scattering calculation for a two-dimensional Hubbard model to study the disappearance of superconductivity at the end of the overdoped region of the cuprate phase diagram. We have found that the decrease in the $d$-wave pairing strength with increasing doping is closely related to a similar decrease in the strength of the $d$-wave spin-fluctuation interaction. The additional effect of impurity scattering, taken into account within a disordered BCS $d$-wave approximation, is found to lead to a further reduction of $T_{c}$ as the doping increases. Hence, in this work, the decrease of $T_{c}(x)$ in the overdoped regime reflects both a decrease in the "clean" transition temperature $T_{c 0}(x)$ due to a reduction in the pairing strength and an increase in the impurity scattering rate with doping. Alternatively, "dirty $d$-wave" models [11] in which $T_{c 0}(x)$ is constant and there is an increase in the Born impurity scattering, starting from a finite doping, to fit the observed $T_{c}(x)$ have proved very useful. However, in this case where $T_{c 0}$ is a constant greater than the maximum $T_{c}$, the required impurity scattering rate for the dirty $d$-wave model will be considerably larger than what we have used.

\section{ACKNOWLEDGMENTS}

The authors would like to thank P. J. Hirschfeld and S. A. Kivelson for their helpful comments. This work was supported by the Scientific Discovery through Advanced Computing (SciDAC) program funded by the U.S. Department of Energy, Office of Science, Advanced Scientific Computing Research and Basic Energy Sciences, and Division of Materials Sciences and Engineering. An award of computer time was provided by the INCITE program. This research used resources of the Oak Ridge Leadership Computing Facility, which is a DOE Office of Science User Facility supported under Contract No. DE- AC05-00OR22725.
[1] Cyril Proust, Etienne Boaknin, R. W. Hill, Louis Taillefer, and A. P. Mackenzie, Heat Transport in a Strongly Overdoped Cuprate: Fermi Liquid and Pure $d$-Wave BCS Superconductor, Phys. Rev. Lett. 89, 147003 (2002).

[2] A. F. Bangura, P. M. C. Rourke, T. M. Benseman, M. Matusiak, J. R. Cooper, N. E. Hussey, and A. Carrington, Fermi surface and electronic homogeneity of the overdoped cuprate superconductor $\mathrm{Tl}_{2} \mathrm{Ba}_{2} \mathrm{CuO}_{6+\delta}$ as revealed by quantum oscillations, Phys. Rev. B 82, 140501(R) (2010).

[3] K. P. Kramer, M. Horio, S. S. Tsirkin, Y. Sassa, K. Hauser, C. E. Matt, D. Sutter, A. Chikina, N. B. M. Schroter, J. A. Krieger et al., Band structure of overdoped cuprate superconductors: Density functional theory matching experiments, Phys. Rev. B 99, 224509 (2019).

[4] Doiron-Leyraud et al., Pseudogap phase of cuprate superconductors confined by Fermi surface topology, Nat. Commun. 8, 2044 (2017).

[5] T. Maier, M. Jarrell, T. Pruschke, and J. Keller, $d$-Wave Superconductivity in the Hubbard Model, Phys. Rev. Lett. 85, 1524 (2000).

[6] M. Jarrell, T. Maier, M. H. Hettler, and A. Tahvildarzadeh, Phase diagram of the Hubbard model: Beyond the dynamical mean field, Europhys. Lett. 56, 563 (2001).

[7] K. S. Chen, Z. Y. Meng, S. X. Yang, T. Pruschke, J. Moreno, and M. Jarrell, Evolution of the superconductivity dome in the two-dimensional Hubbard model, Phys. Rev. B 88, 245110 (2013).

[8] E. W. Huang, D. J. Scalapino, T. A. Maier, Brian Moritz, and T. P. Devereaux, Decrease of $d$-wave pairing strength in spite of the persistence of magnetic excitations in the overdoped Hubbard model, Phys. Rev. B 96, 020503 (2017).

[9] T. A. Maier, M. Jarrell, T. C. Schulthess, P. R. C. Kent, and J. B. White, Systematic Study of $d$-Wave Superconductivity in the 2D Repulsive Hubbard Model, Phys. Rev. Lett. 95, 237001 (2005).
[10] T. A. Maier and D. J. Scalapino, Disappearance of superconductivity in the overdoped cuprates, J. Supercond. Nov. Magn. 33, 15 (2020).

[11] N. R. Lee-Hone, V. Mishra, D. M. Broun, and P. J. Hirschfeld, Optical conductivity of overdoped cuprate superconductors: Application to LSCO, Phys. Rev. B 98, 054506 (2018).

[12] E. Gull, P. Werner, O. Parcollet, and M. Troyer, Continuoustime auxiliary-field Monte Carlo for quantum impurity models, Europhys. Lett. 82, 57003 (2008).

[13] E. Gull, P. Staar, S. Fuchs, P. Nukala, M. S. Summers, T Pruschke, T. Schulthess, and T. Maier, Submatrix updates for the continuous-time auxiliary-field algorithm, Phys. Rev. B 83, 075122 (2011).

[14] Wei Wu, M. S. Scheurer, S. Chatterjee, S. Sachdev, A. Georges, and M. Ferrero, Pseudogap and Fermi-surface topology in the two-dimensional Hubbard model, Phys. Rev. X 8, 021048 (2018).

[15] R. Hlubina, S. Sorella, and F. Guinea, Ferromagnetism in the Two Dimensional $t-t^{\prime}$ Hubbard Model at the Van Hove Density, Phys. Rev. Lett. 78, 1343 (1997).

[16] H. C. Robarts, M. Barthelemy, M. Garcia-Fernandez, J. Li, A. Nag, A. C. Walters, K. J. Zhou, and S. M. Hayden, Anisotropic damping of the spin fluctuations in doped $\mathrm{La}_{2-x} \mathrm{Sr}_{x} \mathrm{CuO}_{4}$ studied by resonant inelastic x-ray scattering, Phys. Rev. B 100, 214510 (2019).

[17] L. J. P. Ament, M. van Veenendaal, T. P. Devereaux, J. P. Hill, and J. van den Brink, Resonant inelastic x-ray scattering studies of elementary excitations, Rev. Mod. Phys. 83, 705 (2011).

[18] S. Wakimoto, K. Ishii, H. Kimura, M. Fujita, G. Dellea, K. Kummer, L. Braicovich, G. Ghiringhelli, L. M. Debeer-Schmitt, and G. E. Granroth, High-energy magnetic excitations in overdoped $\mathrm{La}_{2-x} \mathrm{Sr}_{x} \mathrm{CuO}_{4}$ studied by neutron and resonant inelastic x-ray scattering, Phys. Rev. B 91, 184513 (2015).

[19] D. Meyers, H. Miao, A. C. Walters, V. Bisogni, R. S. Springell, M. d'Astuto, M. Dantz, J. Pelliciari, H. Y. Huang, J. Okamoto, 
D. J. Huang, J. P. Hill, X. He, I. Božović, T. Schmitt, and M. P. M. Dean, Doping dependence of the magnetic excitations in $\mathrm{La}_{2-x} \mathrm{Sr}_{x} \mathrm{CuO}_{4}$, Phys. Rev. B 95, 075139 (2017).

[20] T. A. Maier, A. Macridin, M. Jarrell, and D. J. Scalapino, Systematic analysis of a spin-susceptibility representation of the pairing interaction in the two-dimensional Hubbard model, Phys. Rev. B 76, 144516 (2007).

[21] C. Panagopoulos, J. L. Tallon, B. D. Rainford, T. Xiang, J. R. Cooper, and C. A. Scott, Critical Behavior in the Low-Energy Spin Fluctuations in High- $T_{c}$ Superconductors, J. Supercond. 15, 335 (2002).

[22] Risdiana, T. Adachi, N. Oki, S. Yairi, Y. Tanabe, K. Omori, Y. Koike, T. Suzuki, I. Watanabe, A. Koda, and W. Higemoto, $\mathrm{Cu}$ spin dynamics in the overdoped regime of $\mathrm{La}_{2-x} \mathrm{Sr}_{x} \mathrm{Cu}_{1-y} \mathrm{Zn}_{y} \mathrm{O}_{4}$ probed by muon spin relaxation, Phys. Rev. B 77, 054516 (2008).

[23] M. A. Baqiya, T. Adachi, A. Takahashi, T. Konno, T. Ohgi, I. Watanabe, and Y. Koike, Muon spin relaxation study of the spin correlation in the overdoped regime of electron-doped high- $T_{c}$ cuprate superconductors, Phys. Rev. B 100, 064514 (2019).

[24] K. Kudo, and K. Yamada, Reduction of $T_{c}$ due to Impurities in Cuprate Superconductors, J. Phys. Soc. Jpn. 73, 2219 (2004).

[25] A. A. Abrikosov and L. P. Gorkov, On the theory of superconducting alloys, Sov. Phys. JETP 8, 1090 (1959). 